opposto il corpo sonoro, si potrà seguire il modificarsi di questa curva fino allo spegnersi delle oscillazioni del corista.

Modena, Aprile 1903.

SULLE OPPoste VARIAZIoNi di RESISTENza DEI COHBRER a PEROSSIDO DI PIOMBO PER INFLUENZA DELLE ONDE ELETTRICHE.

Nota del Prof. ERNESTO DRAGO ${ }^{1}$ ).

Nel 1900 il Branly pubblicò una memoria, nella quale espose i risultati di alcune sue ricerche sull' accrescimento di resistenza dei coherer a $\mathrm{PbO}^{2}$ per azione delle onde elettriche. Egli affermò come le sue esperienze lo avevano condotto nell'opinione che l' accrescimento di resistenza dei detti coherer, dipende, al pari della diminuzione da uno stato fisico della membrana isolante interposta fra le particelle. Aschkinass cerca di ricondurre il comportamento del $\mathrm{PbO}^{2}$ a quello dei contatti umidi di conduttori metallici, i quali sotto l'influenza delle onde elettriche aumentano di resistenza.

In una mia memoria precedente ${ }^{2}$ ) ho mostrato come i co. herer a $\mathrm{PbO}^{2}$ ed a $\mathrm{CuS}$ manifestino in certi casi delle dimi. nuzioni di resistenza sotto l'influenza delle onde elettriche.

In seguito ho voluto studiare con maggiore accuratezza tale fenomeno da me constatato per i coherer a $\mathrm{PbO}^{2}$, e nel contempo ho cercato di indagare anche la causa del noto au. mento di resistenza dei detti coherer pel' l'influenza delle onde elettriche. La disposizione sperimentale messa in pratica è stata la seguente. Nel circuito di una pila termoelettrica o di tre elementi normali Raoult, erano inseriti il coherer, un galvanometro a grande resistenza con $i$ rocchetti in serie, astatiz. zato con la durata d'oscillazione di $10^{3}$, ed un interruttore a mercurio.

Ho fatto delle osservazioni col microscopio e con ingrandimenti di 80 o 120 diametri sopra un coherer costituito da

1) Lavoro eseguito nell' Istituto Fisico della R. Unirersità di Catania diretto dal Prof. R. P. Grimaldi.

2) Atti Accademia Gioenia di Catania. Serio 4., Vol. 17. 
una foglia di stagnola incollata sopra un portaoggetti e provvista nel centro di una fenditura di larghezza variabile nell'ordine dei millimetri o frazioni di millimetro. Su tale fenditura mettevo la polvere di $\mathrm{PbO}^{2}$ e poi con una punta d'ago da cucire costruivo ponti di detta polvere con o senza interruzione secondo il bisogno delle esperienze. L' esame microscopico veniva anche esteso ai coherer di forma ordinaria, il cui vetro era bucato fra gli elettrodi per la possibile costruzione o interruzione dei ponti.

Sin dal principio delle esperienze ho potuto constatare in certi casi la formazione di ponti di $\mathrm{PbO}^{3}$ e la conseguente conduttività al galvanometro, collegando direttamente un polo del secondario di un piccolo rocchetto di Ruhmkorff, messo in azione da un elemento di Cupron, con un estremo del coherer. In seguito ho eseguito le ricerche servendomi dell' azione dell'oscillatore di Lodge, posto a circa un metro di distanza del coherer, ed ho trovato confermati i risultati di cui ho fatto cenno.

La formazione dei ponti era evidentissima quando si facevano agire le onde elettriche sul coherer, mentre il circuito di questo era aperto in un punto speciale che determinavo per tentativi.

Poichè le scintille nella polvere di $\mathrm{PbO}^{\text {s }}$ avevano un' in. fluenza perturbatrice sui fenomeni cercai di eliminarle nel modo migliore che mi fu possibile.

Per escludere poi l'opinione di Sundorph, il quale crede che l'aumento di resistenza dei coherer a $\mathrm{PbO}$ per azione delle onde elettriche sia prodotto dalla trasformazione di detta sostanza nel $\mathrm{PbO}$ cattivo conduttore e per cercare la vera causa del detto aumento di resistenza istitui :

$1^{0}$ Ricerche fotografiche

$2^{0}$ Esperienze con elettrodi di natura diversa

$3^{0}$ Esperienze con il $\mathrm{PbO}^{2}$ nell' aria rarefatta

$4^{0}$ Ricerche chimiche

$5^{0}$ Esperienze con ponti sottilissimi di $\mathrm{PbO}^{2}$

$6^{\circ}$ Esperienze con polveri metalliche.

Dalle mie ricerche ed esperienze sono indotto a concludere che: 
$\mathrm{I}^{0}$ I coherer a $\mathrm{PbO}^{2}$ possono non solamente manifestare degli aumenti di resistenza sotto l'infuenza delle onde elettriche, ma anche delle DIMINuzion.

$2^{0} L^{\prime}$ aumento di resistenza dei coherer a $\mathrm{PbO}^{2}$ si ottiene quando $i$ medesimi sono sottoposti all' azione di onde intense, mentre la diminuzione di resistenza dei detti coherer si presenta quando questi sono sottoposti all' azione di onde debolissime, e cresce con il tempo d'azione delle medesime.

$3^{0}$ La causa dell' aumento di resistenza dei coherer a $\mathrm{PbO}^{\mathrm{a}}$ risiede nella distruzione dei ponti conduttori precedentemente stabiliti mentre la causa della diminuzione va ascritta alla chiusura di intermzioni di ponti conduttori. $L$ ' urto meccanico sul coherer rompe $i$ ponti ed annulla la conauttivita stabilita precedentemente delle onde eleltriche.

$4^{0}$ Perché tali chiusure e distruzioni di ponti possono manifestarsi con nettezza $\dot{e}$ necessario costruire dei ponti elementari con particelle esilissime di $\mathrm{PbO}^{2}$. Se si eseguono delle ricerche con grani grossi di polvere possono venire mascherate le osservazioni per le quali si traggono le pre. cedenti conclusiani. Difatti interviene allora il disgrega. mento dei granelli di polvere in minutissimi corpuscoli, il quale ha sui grani la stessa azione di uno scuotimento.

$5^{0}$ Nelle stesse condizioni in cui i coherer a $\mathrm{PbO}^{2}$ ma. nifesiano una diminuzione della loro resistenza eletrica, $i$ coherer stratiformi metallici sono poco sensibili, e la for. mazione di catene conduttrici non sembra essere causata essenzialmente da scintille fra grano e grano di polvere, ed avviene con movimenti poco sensibili e con piccolissime distanze fra $i$ grani.

Senza bisogno di ricorrere alla teoria di Bose, la quale presenta qualche lacuna, e ricordando le esperienze di Tommasina, il quale, ripetendo le ricerche sui coherer di potassio e sodio immersi nel petrolio ed esposti all' azione delle onde elettriche, trova per $\mathrm{i}$ medesimi degli aumenti di resistenza che spiega benissimo con la teoria della coerenza, io credo che le mie presenti ricerche possono fare entrare il $\mathrm{PbO}^{2}$ nella stessa teoria. 
Oramai mi sembra abbastanza evidente perchè nelle esperienze di Branly «sull' accrescimento di resistenza dei radioconduttori » toccando con un polo della macchina Wimshurst il tubo a $\mathrm{PbO}^{2}$ la resistenza del medesimo cresceva fino a $10000 \mathrm{ohm}$. Io ho ripetuto le dette esperienze ed ho notato che toccando con un polo della macchina Wimshurst un estremo del coherer stratiforme a $\mathrm{PbO}^{2}$, i ponti di polvere precedentemente stabiliti si rompevano, mentre adoperando limatura metallica nelle stesse condizioni i ponti precedentemente aperti si chiudevano. La polvere di $\mathrm{PbO}^{*}$ è formata da grani complessi costituenti alla loro volta leggieri ed esili corpuscoli, ed è quindi evidente che soltanto un azione debolissima può mettere in evidenza il fenomeno della coerenza.

\section{LIBRI NUOVI}

\section{UR THEORIE DER MTKROSKOPISGEEN BILDEAZEUGUNG,} von VICTOR GRÜNBERG

Ptofessar an der. Landes-Oberredschule in Znaim.

(Leipzig, Verlag ron J. A. Barth, 1903. -- Un volumetto di pag. 90).

L'Autore, con l'intento di agevolare l'intelligenza delle cose esposte, segue, salvo alcuni opportuni cambiamenti, il metodo tenuto dal Dippel nel suo Manúale di microscopia generale; e al capitolo, che tratta della teoria della formazione dell' immagine microscopica, premette una serie di considerazioni e di teoremi riguardanti la refrazione e la formazione delle immagini in generale.

L' ordinamento della materia apparisce dai titoli dei capitoli che sono questi: Refrazione in una superficie sferica; Lenti infinitamente sottili e sistemi di lenti; Equazioni di Töpler; Punti cardinali di un sistema; Derivazione delle cinque più importanti equazioni per un sistema ottico; Intensità luminosa; Teoria della formazione della immagine microscopica.

Dott. T. GIGLI. 